\title{
THE MIXING LENGTH RATIO, EDDY DIFFUSIVITY, AND ACOUSTIC WAVES
}

\author{
Kwing L. Chan \\ Applied Research Corp. \\ Landover, Maryland, U. S.A. \\ and \\ Sabatino Sofia \\ Center fo Solar and Space Research, Yale University \\ New Haven, Connecticut, U.S.A.
}

Many processes in the convection zone of a star affect the evolution and the atmospherio diagnostics. Here, a progress report is given on our numerical study of some convection related phenomena. The numerical results are obtained by solving the Navier stokes equations in a three dimensional rectangular domain. The units are chosen such that the initial temperature, pressure, density, and the depth of the domain are all normalized to 1 .

\section{A. The mixing length ratio}

The mixing length theory relates the convective (enthalpy) flux $F_{C}$ to the envelope structure quite well (Chan and Sofia 1987). For efficient convection that occurs in deep convective regions, the numerical results are compatible with a mixing length ratio of 2.1 . The mixing length theory fails to address the significance of the flux of kinetic energy $F_{K E}$ (see Figure 1). $F_{K E}$ is negative and has a magnitude comparable to the total flux. These results are qualitatively similar to those of two dimensional computations (Hurlburt et. al. 1984).

\section{B. Diffusive action of the convective turbulence}

The convective turbulence tend to dissipate large scale shears (wave length i > pressure scale height $H$ ). The rate of dissipation is approximately proportional to $i^{-2}$, and the effective kinematic viscosity turns out to be about $\frac{1}{4} \mathrm{rms}\left(\mathrm{V}_{\mathrm{z}}\right) \mathrm{H}( \pm 50 \%)$ where $\mathrm{rms}\left(\mathrm{V}_{\mathrm{z}}\right)$ is the root-mean-square vertical velocity. The convective turbulence also diffuses large scale temperature perturbations. The effective diffusivity is approximately $\frac{1}{3} \mathrm{rms}\left(\mathrm{V}_{\mathrm{z}}\right) \mathrm{H}$. The temperature perturbation excites acoustic oscillations right away (see next section).

\section{Acoustic waves in the convection zone}

Finite-amplitude oscillations oo-exist with the convective turbulence. The frequencies of the modes are in agreement with the acoustic frequencies obtained by eigenvalue analysis (see Figure 2).

This research is partially supported by the NSF (AST-8504398). 


\section{REFERENCES}

Chan, K. L. and Sofia, S. 1987, SCIENCE, 235, 465.

Hurlburt, N. E., Toomre, J., and Massaguer, J. M. 1984, Ap. J., 282 , 557.

\section{Eigure 1.}

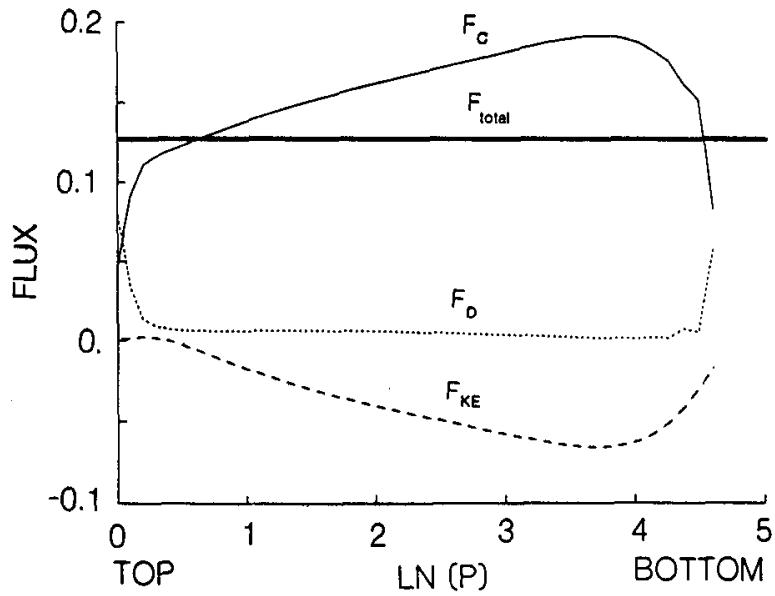

An example of the distributions of fluxes versus depth, ln(pressure). The diffusive flux (dotted curve) is very small except near

boundaries. The flux of kinetic energy (dashed curve) is downward and its magnitude reaches about $50 \%$ of the total flux (thick solid curve). This makes the enthalpy flux (thin solid curve) nonuniform.

\section{Eigure 2.}

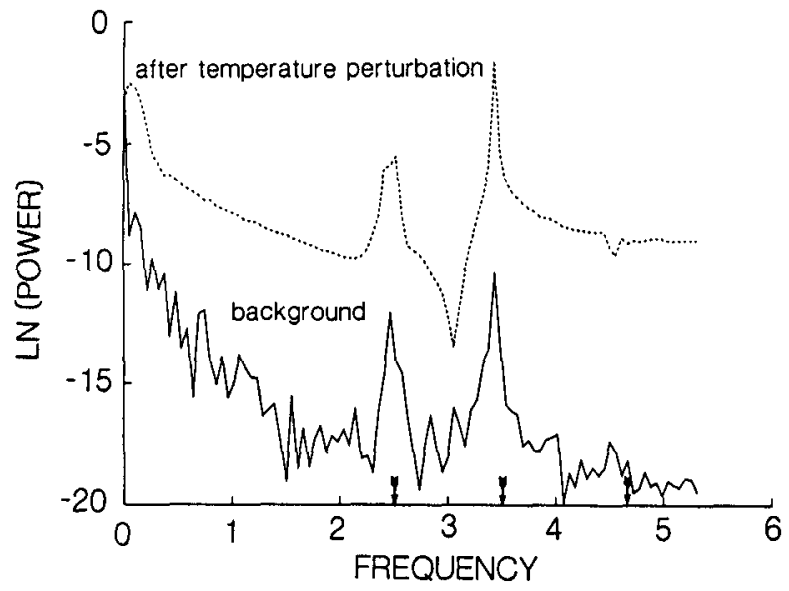

Frequency spectra showing peaks at acoustic frequencies predicted analytically (arrows). The dotted curve is for the case with temperature perturbation, and the solid curve is for the background state. 